\title{
The influenc of hydrolyzed and non-hydrolyzed linden inflorescence (Tilia cordata) extract on metabolic and transcriptomic profile in rat liver
}

\author{
J. Wilczak¹, M. Łój, A. Prostek, D. Kamola, H. Kosińska, and M. Jank \\ Warsaw University of Life Sciences-SGGW, Faculty of Veterinary Medicine, Nowoursynowska 159, 02-776 Warsaw
}

KEY WORDS: flavonoids, quercetin, Tilia cordata, transcriptomic, antioxidant, liver, rats

Received 5 October 2012

Revised 4 March 2013

Accepted 20 March 2013

${ }^{1}$ Corresponding author: e-mail: jacek_wilczak@sggw.pl

\begin{abstract}
The extract from linden inflorescence is one of the pharmacognostic resources which properties are associated with the presence of flavonoids (mainly: quercetin, kaempferol, acacetin glycosides and tiliroside). Flavonoids belong to polyphenols that may play a significant role in the dietoprophylaxis of civilization diseases. These compounds have many different mechanisms of action, but most important seem to be their antioxidant properties. The aim of the present study was to investigate the influence of linden extract on a) gene expression in rat liver and determine the difference in gene expression depending on the use of hydrolyzed or non-hydrolyzed linden extract, and b) antioxidant parameters of liver tissue. Rats were fed the diet containing hydrolyzed and non-hydrolyzed extract from linden inflorescence (Tilia cordata). The administration of hydrolyzed extract increased more than two-fold the level of quercetin in rats liver when compared to non-hydrolyzed extract. The transcriptomic study performed using microarray technology revealed 344 probes regulated by linden extract and 187 probes differentiating the action of hydrolyzed from nonhydrolyzed linden extracts. The most important molecular functions of regulated genes were as follows: defense and immunity, transporter, receptor, ion channel, oxidoreductase, cytoskeletal protein and cell adhesion molecule. Among most important biological processes identified were immunity and defense, transport, homeostasis and lipid, fatty acid and steroid metabolism. The analysis of oxidative status in rats liver together with the analysis of liver transcriptomic profile suggest that the antioxidant activity of hydrolyzed linden extract is higher that the non-hydrolyzed and occurs on the level of gene expression.
\end{abstract}

\section{Introduction}

Extract from linden inflorescence (Tilia cordata) is one of the pharmacognostic resources which properties are associated with the presence of flavonoids. The main flavonoids present in linden flower are quercetin and kaempherol (Kohlmunzer 1933; Sadowska, 2003). Flavonoids belong to polyphenols which may play a significant role in the dietoprophylaxis of civilization diseases. These compounds have many different mechanisms of action, but most important seem to be their antioxidant properties (Hodek et al., 2002).

Flavonoon form which have lower molecular weight and hydrophobic properties. The aglycones of flavonoids can be transported across the 
membranes of enterocytes viaapassive transport.

This is why hydrolysis of water linden extract results in better bioavailability of flavonoids when they are given orally (Aherne and O'Brien, 2002). Quercetin is a powerful antioxidant that is widely distributed in edible plants, mainly as glycosides such as rutin. The results of studies shown that rutin is absorbed more slowly than quercetin because it must be hydrolyzed by the cecal microflora, whereas quercetin is absorbed from the small intestine (Crespy et al., 2002; Manach et al., 1997). Dihal et al. (2006) revealed that quercetin, but not rutin inhibits azoxymethane-induced colorectal carcinogenesis in F344 rats. The aim of the present study was to investigate if rats which diets were enriched with hydrolyzed and non-hydrolyzed linden extracts have different blood parameters, liver transcriptomic profiles and some parameters of liver tissue antioxidant status.

\section{Material and methods}

\section{Animals}

Spraque-Dawley male rats at the age of 8 weeks were divided into three groups (number of rats in each group was 10). One was control group receiving standard diet (Labofeed D, Poland) (Table 1) whereas two experimental groups were receiving the diet enriched with $1 \%$ nonhydrolyzed water extract from linden inflorescence or $1 \%$ water extract from linden inflorescence hydrolyzed using $\mathrm{HCl}$. Water and feed were provided ad libitum.

Table 1. The composition of standard diet fed for 6 weeks to 8-weekold rats. The diets were enriched with $1 \%$ nonhydrolyzed or hydrolyzed water extract from linden inflorescence, depending on the group

\begin{tabular}{lc}
\hline Ingredients & Content, \% \\
\hline Protein & 22.5 \\
Lysine & 1.2 \\
Crude fat & 5 \\
Crude fibre & 4.5 \\
Crude ash & 6.5 \\
Calcium & 0.9 \\
Phosphorus & 0.7 \\
& \\
Vitamin A & $15.000 \mathrm{IE} \cdot \mathrm{kg}^{-1}$ \\
Vitamin $\mathrm{D}_{3}$ & $600 \mathrm{IE} \cdot \mathrm{kg}^{-1}$ \\
Vitamin E & $75 \mathrm{mg} \cdot \mathrm{kg}^{-1}$ \\
Copper & $5 \mathrm{mg} \cdot \mathrm{kg}^{-1}$ \\
\hline
\end{tabular}

The study lasted 6 weeks. After this period of time animals were euthanized with isoflurane inhalation, then livers were collected during autopsy.

\section{Microarrays}

Liver samples were frozen with liquid nitrogen immediately after collection and stored at $-80^{\circ} \mathrm{C}$ until extraction. Total RNA was extracted with Total RNA kit (A\&A BIOTECHNOLOGY, Poland) following the manufacturer's recommended protocol. Subsequently, potential genomic DNA contamination was eliminated using Deoxyrybonuclease I Amplification Grade (Sigma, USA) and RNeasyMinElute Cleanup Kit (Qiagen, Germany). RNA quantity and quality was measured using NanoDrop ND1000 (NanoDrop Technologies, USA) and Bioanalyzer (Agilent Technologies, USA).

Afterwards, samples were pooled as follows: 1) combined RNA extracted from 5 rats which received diet enriched with non-hydrolyzed Tilia cordata extract, and 2) combined RNA extracted from 5 rats which received diet enriched with hydrolyzed Tilia cordata extract.

Preparation of cDNA was carried out using SuperScript Plus Indirect cDNA Labeling Kit (Invitrogen, USA). cDNA was labeled with Alexa555 and Alexa647. Every pool was labeled 6 times (3 times with Alexa555 and with Alexa 647 dye swap quality control). Samples were analyzed on cDNA microarrays Array-Ready Oligo Set ${ }^{\mathrm{TM}}$ for the Rat Genome Version 3.0 (Operon, Germany). Hybridization was carried with an automated hybridization system HybArray12 (PerkinElmer, USA). Microarrays were scanned using DNA ScanArray HT (PerkinElmer, USA).

A statistical analysis was performed using linear model that was described by Smyth (2004). Student's t-test was used to find genes with altered expression between research groups.

\section{The antioxidant parameters}

The quercetin concentration in liver homogenates we assayed using HPLC method with electrochemical detection. To express liver tissue antioxidant status the activity of superoxide dismutase (SOD) and total antioxidant status (TAS) were measured using Randox test. To estimate the ability of lipids in hepatocytes cell membranes to oxidation the sum of tiobarbituric acid reactive substances (TBARS) were measured, based on the following method: a final reaction mix containing liver tissue homogenates, $0.8 \%$ SDS, $10 \%$ acetic acid, and $0.17 \%$ thiobarbituric acid were incubated for $1 \mathrm{~h}$ at $100^{\circ} \mathrm{C}$, centrifuged, and then absorbance values were measured at $530 \mathrm{~nm}$. Values were referred to a calibration curve of 1,1,3,3-tetraethoxypropane. 


\section{Statistical evaluation}

The results were statistically evaluated using GraphPadPrism $^{\mathrm{TM}}$ version 5.00 software (GraphPad Software, Inc., La Jolla, CA, USA). Comparisons between two groups were evaluated using Student's $\mathrm{t}$-test. A probability: $\mathrm{P} \leq 0.05$ was regarded as significant.

\section{Results}

\section{The transcriptomic profile of livers from rats receiving hydrolyzed and non-hydrolyzed linden extract}

The statistical analysis revealed 532 differentially regulated genes $(\mathrm{P}<0.05)$ in the livers of rats receiving hydrolyzed and non-hydrolyzed linden extract groups. Among them there were 43 genes regulated at the highest level of significance $(\mathrm{P}<0.01)$ (data not shown).

The analysis of regulated genes ontology and function conducted using Pathway Studio Software showed that among differentially expressed genes there were 27 ones regulating the generation of reactive oxygen species (ROS) (Table 2). This analysis revealed also that the regulated genes differentiating the action of hydrolyzed and non-hydrolyzed linden extract groups regulate the expression of phase II detoxifying enzymes such as NQO1 (Figure 1) and $\mathrm{HO}^{-1}$ (Figure 2).

Pathway Studio software allows also the identification the factors (or proteins) which are responsible for the regulation of identified genes expression (so called expression targets). In our study the following proteins were identified as factors which regulate the expression of highest numbers (over 10) of genes considered as regulated ones: prostaglandin-endoperoxide synthase 2 (PTGS2), vascular endothelial growth factor A (VEGFA), tumor necrosis factor (TNF superfamily, member 2) (TNF), interleukin 6 (interferon, beta 2) (Il6), cyclin D1 (CCND1), nitric oxide synthase 2 , inducible (NOS2), interleukin 8 (Il8), matrix metallopeptidase 9 (MMP9), interleukin 2 (Il2), chemokine (C-C motif) ligand 2 (CCL2), matrix metallopeptidase $1(M M P 1)$, B-cell CLL/lymphoma 2 (BCL2), BCL2-associated $\mathrm{X}$ protein $(B A X)$,

Table 2. The list of differentially expressed genes involved in reactive oxygen species generation in liver of rats fed with the diets enriched with $1 \%$ hydrolyzed or $1 \%$ non-hydrolyzed linden extract for 6 weeks weeks

\begin{tabular}{|c|c|c|c|c|}
\hline Gene symbol & RefSeq & Description & $\log \mathrm{FC}$ & P-value \\
\hline PTK2 & NM_013081.1 & PTK2 protein tyrosine kinase 2 & -0.514 & 0.028 \\
\hline$P R L$ & NM_012629.1 & Prolactin & -0.659 & 0.015 \\
\hline MYC & NM_012603.2 & v-myc myelocytomatosis viral oncogene homolog (avian) & -0.57 & 0.029 \\
\hline MAP3K5 & XM_218780.2 & Mitogen-activated protein kinase kinase kinase 5 & 0.56 & 0.023 \\
\hline EDNRA & NM_012550.1 & Endothelin receptor type A & 0.654 & 0.014 \\
\hline CCL20 & NM_019233.1 & Chemokine (C-C motif) ligand 20 & -0.623 & 0.017 \\
\hline SERPINE1 & NM_012620.1 & Serpin peptidase inhibitor. clade $\mathrm{E}$ (plasminogen activator inhibitor type 1) & -0.713 & 0.029 \\
\hline PAK1 & NM_017198.1 & p21 protein (Cdc42/Rac)-activated kinase 1 & -0.412 & 0.027 \\
\hline MTOR & NM_019906.1 & Mechanistic target of rapamycin (serine/threonine kinase) & -0.762 & 0.011 \\
\hline ATM & XM_236275.3 & Ataxia telangiectasia mutated & -0.409 & 0.048 \\
\hline TRPV1 & NM_031982.1 & Transient receptor potential cation channe, subfamily V, member 1 & -0.471 & 0.034 \\
\hline CXCL3 & NM_138522.1 & Chemokine (C-X-C motif) ligand 3 & -0.448 & 0.031 \\
\hline SYK & NM_012758.1 & Spleen tyrosine kinase & 0.488 & 0.044 \\
\hline SEMA3A & NM_017310.1 & $\begin{array}{l}\text { serpin peptidase inhibitor, clade } E \text { (nexin. plasminogen activator inhibitor } \\
\text { type 1), member } 1\end{array}$ & 0.494 & 0.037 \\
\hline NR3C2 & NM_013131.1 & Suclear receptor subfamily 3 , group C, member 2 & -0.546 & 0.047 \\
\hline TRIB3 & NM_144755.1 & Tribbles homolog 3 (Drosophila) & -0.471 & 0.041 \\
\hline$C P$ & NM_001270961.1 & Ceruloplasmin (ferroxidase) & 0.541 & 0.033 \\
\hline CKMT1B & NM_001012738.1 & Creatine kinase, mitochondrial 1B & -0.605 & 0.016 \\
\hline$A D A$ & NM_130399.2 & Adenosine deaminase & -0.659 & 0.049 \\
\hline$H P$ & NM_012582.2 & Haptoglobin & 0.519 & 0.043 \\
\hline MAP3K7 & XM_232855.3 & Mitogen-activated protein kinase kinase kinase 7 & -0.543 & 0.048 \\
\hline FDX1 & NM_017126.1 & Ferredoxin 1 & -0.541 & 0.032 \\
\hline GGPS1 & NM_001007626.1 & Geranylgeranyl diphosphate synthase 1 & -0.465 & 0.026 \\
\hline HSP90AB1 & NM_001004082.2 & Heat shock protein 90kDa alpha (cytosolic), class B member 1 & 0.564 & 0.028 \\
\hline ACP1 & NM_021262.2 & Acid phosphatase 1. soluble & -0.679 & 0.041 \\
\hline CYP2A13 & NM_012692.1 & Cytochrome P450, family 2, subfamily A, polypeptide 13 & -0.695 & 0.036 \\
\hline CTRL & NM_054009.1 & Chymotrypsin-like & -0.807 & 0.024 \\
\hline
\end{tabular}


FBJ murine osteosarcoma viral oncogene homolog (FOS), interferon, gamma (IFNG), tumor protein p53 (TP53), cyclin-dependent kinase inhibitor 1B (p27, Kipl) (CDKN1B), interleukin 10 (Il10), bone gamma-carboxyglutamate (gla) protein $(B G L A P)$.

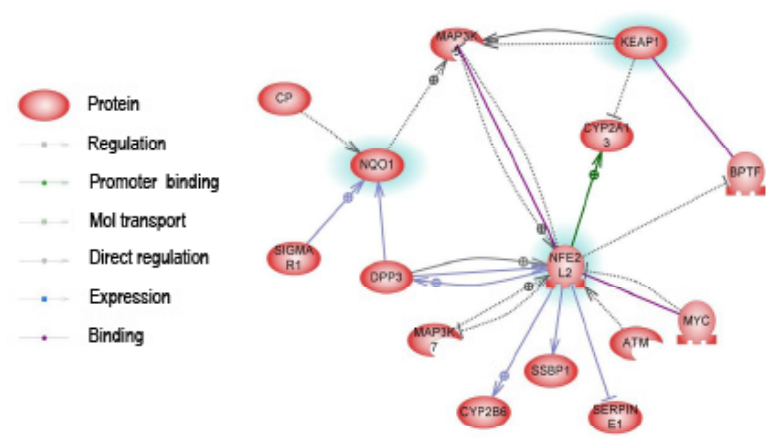

Figure 1. The relations between Nrf2 (NFE2L2), Keap1, NQ01 proteins and proteins encoded by genes differentially regulated in livers of rats fed for 6 weeks with the diets enriched with $1 \%$ hydrolyzed or $1 \%$ non-hyrolyzed linden extracts

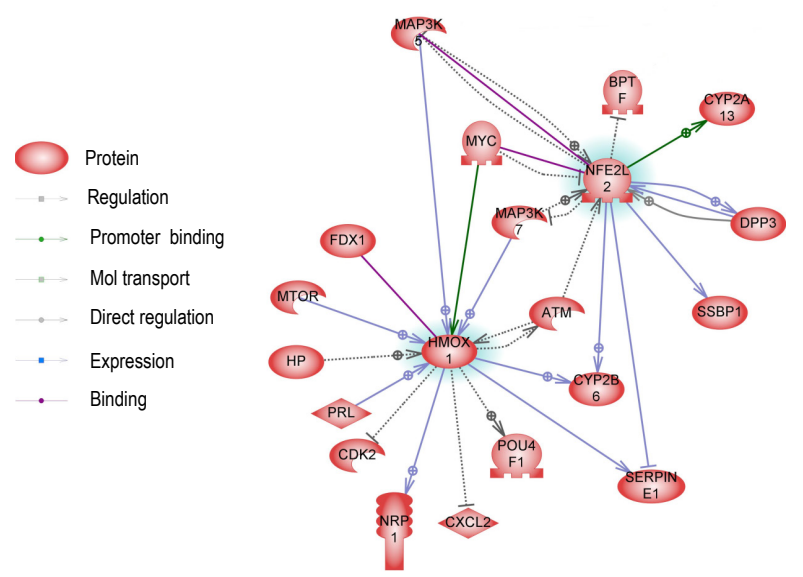

Figure 2. The relations between Nrf2 (NFE2L2), HO-1 proteins and proteins encoded by genes differentially regulated in livers of rats fed for 6 weeks with the diets enriched with $1 \%$ hydrolyzed or $1 \%$ nonhydrolyzed linden extracts

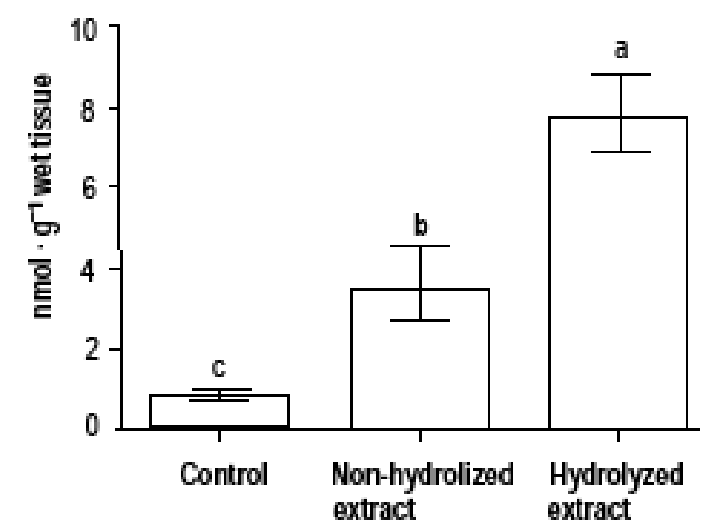

Figure 3. Quercetin content in liver tissue of rats fed for 6 weeks with standard diet and diets enriched with $1 \%$ hydrolyzed or $1 \%$ nonhydrolyzed linden extracts; bars with different letters represents the level of significant $P \leq 0.05$

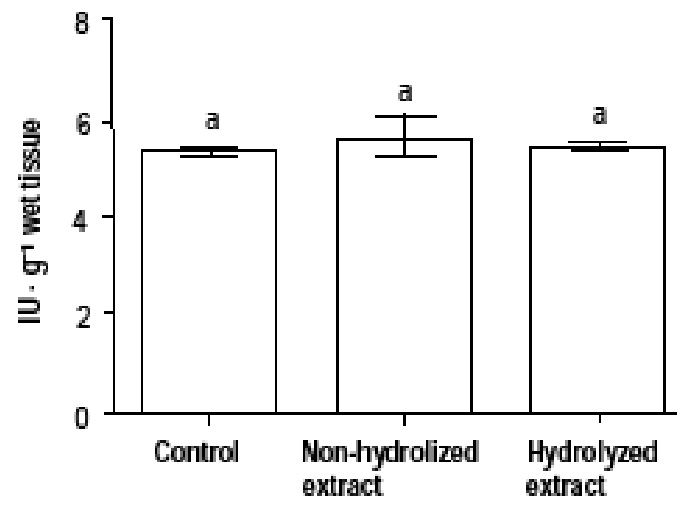

Figure 4. Activity of superoxide dismutase (SOD) in liver tissue of rats fed for 6 weeks with standard diet and diets enriched with 1\% hydrolyzed or $1 \%$ non-hydrolyzed linden extracts; bars with different letters represents the level of significant $P \leq 0.05$

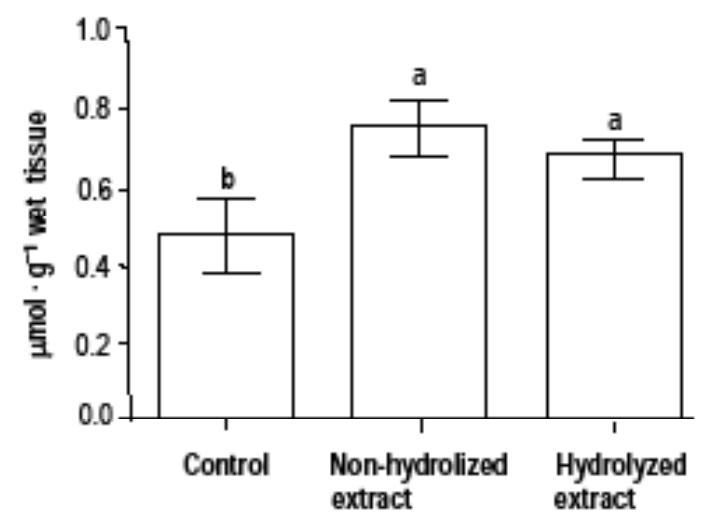

Figure 5. Level of total antioxidant status (TAS) in liver tissue of rats fed for 6 weeks with standard diet and diets enriched with $1 \%$ hydrolyzed or $1 \%$ non-hydrolyzed linden extracts; bars with different letters represents the level of significant $\mathrm{P} \leq 0.05$

\section{Antioxidant action of hydrolyzed and non-hydrolyzed linden extract}

The 6 weeks of the rat diet supplementation with hydrolyzed and non-hydrolyzed linden extract resulted in the highest concentration of quercetin in the liver of rats receiving the hydrolyzed one $(\mathrm{P}<0,05)$. We were also able to identify some traces of quercetin in the liver of control rats which could be a result of its presence in the ingredients used for rat feed production, mainly grains. The acid hydrolysis of linden inflorescence did not influence significantly the activity of superoxide dismutase (SOD). The Total Antioxidant Status (TAS) describes the total antioxidant properties of the tissue (in this case liver) and it was increased by the two investigated extracts, when compared to control rats. The TBARS value is a parameter describing the level of the fatty acid oxidation and it was the highest in the control, nonsupplemented group. The lowest TBARS were observed in the group of rats receiving hydrolyzed linden extract (Figure 6). 


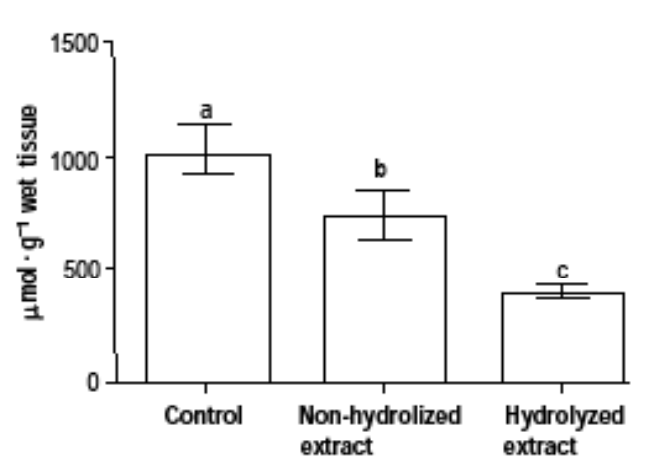

Figure 6. Level of the sum of tiobarbituric acid reactive substances (TBARS) in liver tissue of rats fed for 6 weeks with standard diet and diets enriched with $1 \%$ hydrolyzed or $1 \%$ non-hydrolyzed linden extracts; bars with different letters represents the level of significant $P \leq 0.05$

\section{Discussion}

The results of the present study revealed that genes which were differentially regulated in livers of rats receiving the diet with hydrolyzed or nonhydrolyzed water linden flower extracts are related with generation of reactive oxygen species (ROS) (Table 2). One of the main components of this extract is quercetin that occurs in plants mainly as a glycoside - rutoside. Zerina et al. (2012) have suggested that quercetin may be used to minimize oxidative stress via reducing the generation of ROS. This effect was due to modulating expression of antioxidant genes in A549 cells. Kaempferol is another component of linden flower which also has strong antioxidant properties. Hong et al. (2009) shown that pretreatment of PC12 cells with kaempferol reduced intracellular generation of reactive oxygen species (ROS) in response to $\mathrm{H}_{2} \mathrm{O}_{2}$ and strongly elevated cell viability.

The use of advanced gene ontology analysis tools (Pathway studio) gives the unique possibility of identification of factors (proteins) which regulate the expression of genes identified transcriptomic studies. In the present study we were able to identify proteins for which our regulated genes are the expression targets. The analysis revealed that many our differentially regulated genes were expression targets of proteins involved in inflammation process. Among them were tumor necrosis factor (TNF superfamily, member 2) (TNF) and nitric oxide synthase 2 (NOS2). It is known that quercetin has anti-inflammatory properties through its ability to inhibit nitric oxide production and iNOS expression (Ortega et al., 2010). Also other expression targets such as I16, Il8, I12, IL10, TNF are important for the inflammation process. It is known that phase II detoxifying enzymes, such as $\mathrm{NAD}(\mathrm{P}) \mathrm{H}$ :quinone oxidoreductase (NQO1), glutathione S-transferase (GST), heme oxygenase (HO-1) and nitric oxide synthase (iNOS), contain specific nucleotide sequences - in particular, an antioxidant-responsive element/electrophile-responsive element (ARE/EpRE) - in their gene promoters that contribute to the protection of cells against carcinogens and oxidative stress (Chen and Kong, 2004). There are several molecules proposed as potential modulators of ARE/EpRE. Among them nuclear factor-E2-related factor 2 (Nrf2) has been repeatedly proven to be a strong activator of ARE-mediated gene expression. The study conducted by Weng et al. (2011) shown that quercetin could be beneficial for the prevention of environmental oxidant-induced liver damage. One of the proposed mechanisms of this action was enhancing Nrf2 DNA-binding activity. Another author revealed that quercetin activated Nrf2-ARE-mediated NQO1 transactivation not only through enhanced the steady-state level of Nrf2 at both transcriptional and posttranslational levels, but also reduced the steady-state level of Keap1 through 26S proteasome-independent degradation (Tanigawa et al., 2007). The Figure 1 presents relations between Nrf2 (NFE2L2), Keap1, NQO1 proteins and proteins encoded by genes regulated in our study. The experiments conducted by Granado-Serrano et al. (2012), Tanigawa et al. (2007) and Weng et al. (2011) were performed in human hepatoma cells (HepG2). Our study was performed in vivo and did not check the influence flavonoids in aglycone forms (like above mentioned) but was focused on the differences between flavonoids given in aglycone and glycoside forms. This may be the reason why our results did not show direct changes in expression of Nrf2 (NFE2L2), Keap1 and NQO1, but indicate only changed expression of genes regulated by these three proteins. In our study we were able to identify the 12 genes which were expression targets of NQO1 (Table 3). It may be that the regulation of some genes in our study results from the changed activity of Nrf2 (NFE2L2), Keap1 and NQO1 on protein level and not their changed expression on gene level.

The next important phase II detoxifying enzyme is heme oxygenase (HO-1). Growing data indicate that HO-1 plays a key role in defense mechanisms against oxidative damages (Nakahira et al., 2003). HO- 1 is one of the target genes of transcription factor - Nrf2. Many studies show that changes in expression of HO-1 are related with action of flavonoids, often quercetin and kaempferol. Liu et al. (2012) have suggested that quercetin attenuates ethanol-induced oxidative stress through a pathway which HO-1 upregulation. The second most abundant active substance in linden inflorescence, 
Table 3. The list of differentially regulated genes in liver of rats fed for 6 weeks with the diets enriched with $1 \%$ hydrolyzed or $1 \%$ non-hydrolyzed linden extracts being expression targets of NQO1

\begin{tabular}{|c|c|c|c|c|}
\hline Gene symbol & RefSeq & Description & $\log \mathrm{FC}$ & P-value \\
\hline MAP3K5 & XM_218780.2 & Mitogen-activated protein kinase kinase kinase 5 & 0.56 & 0.023 \\
\hline MYC & NM_012603.2 & v-myc myelocytomatosis viral oncogene homolog (avian) & -0.57 & 0.029 \\
\hline MAP3K7 & XM_232855.3 & $\begin{array}{l}\text { Mitogen-activated protein kinase ki } \\
\text { nase kinase } 7\end{array}$ & -0.543 & 0.048 \\
\hline ATM & XM_236275.3 & Ataxia telangiectasia mutated & -0.409 & 0.048 \\
\hline CYP2B6 & NM_198733.1 & cytochrome P450. family 2, subfamily B, polypeptide 6 & -0.448 & 0.048 \\
\hline SERPINE1 & NM_012620.1 & Serpin peptidase inhibitor, clade $\mathrm{E}$ (plasminogen activator inhibitor type 1) & -0.713 & 0.029 \\
\hline$C P$ & NM_001270961.1 & Ceruloplasmin (ferroxidase) & 0.541 & 0.033 \\
\hline SIGMAR1 & NM_030996.1 & Sigma non-opioid intracellular receptor 1 & -0.481 & 0.019 \\
\hline BPTF & XM_003750948.1 & Bromodomain PHD finger transcription factor & 0.385 & 0.05 \\
\hline CYP2A13 & NM_012692.1 & Cytochrome P450. family 2, subfamily A, polypeptide 13 & -0.695 & 0.036 \\
\hline SSBP1 & NM_183328.2 & Single-stranded DNA binding protein 1 & -0.488 & 0.019 \\
\hline DPP3 & NM_053748.1 & Dipeptidyl-peptidase 3 & -0.414 & 0.048 \\
\hline
\end{tabular}

Table 4. The list of differentially regulated genes in liver of rats fed for 6 weeks with the diets enriched with $1 \%$ hydrolyzed or $1 \%$ non-hydrolyzed linden extracts being expression targets of $\mathrm{HO}-1$

\begin{tabular}{lllcl}
\hline Gene symbol & RefSeq & Description & $\operatorname{logFC}$ & P-value \\
\hline PRL & NM_012629.1 & Prolactin & -0.659 & 0.015 \\
MYC & NM_012603.2 & V-myc myelocytomatosis viral oncogene homolog (avian) & 0.57 & 0.029 \\
MAP3K5 & XM_218780.2 & Mitogen-activated protein kinase kinase kinase 5 & 0.56 & 0.023 \\
CDK2 & NM_199501.1 & Cyclin-dependent kinase 2 & -0.501 & 0.017 \\
SERPINE1 & NM_012620.1 & Serpin peptidase inhibitor, clade E (plasminogen activator inhibitor type 1) & -0.713 & 0.029 \\
MTOR & NM_019906.1 & Mechanistic target of rapamycin (serine/threonine kinase) & -0.762 & 0.011 \\
ATM & XM_236275.3 & Ataxia telangiectasia mutated & -0.409 & 0.048 \\
CXCL3 & NM_138522.1 & Chemokine (C-X-C motif) ligand 3 & -0.448 & 0.031 \\
NRP1 & NM_145098.2 & Neuropilin 1 & -0.591 & 0.01 \\
HP & NM_012582.2 & Haptoglobin & 0.519 & 0.043 \\
MAP3K7 & XM_232855.3 & Mitogen-activated protein kinase kinase kinase 7 & -0.543 & 0.048 \\
FDX1 & NM_017126.1 & Ferredoxin 1 & -0.541 & 0.032 \\
BPTF & XM_003750948.1 & Bromodomain PHD finger transcription factor & 0.385 & 0.05 \\
CYP2A13 & NM_012692.1 & Cytochrome P450, family 2, subfamily A, polypeptide 13 & -0.695 & 0.036 \\
POU4F1 & XM_341372.2 & POU class 4 homeobox 1 & -0.362 & 0.044 \\
SSBP1 & NM_183328.2 & Single-stranded DNA binding protein 1 & -0.488 & 0.019 \\
CYP2B6 & NM_198733.1 & Cytochrome P450, family 2, subfamily B, polypeptide 6 & -0.448 & 0.048 \\
DPP3 & NM_053748.1 & Dipeptidyl-peptidase 3 & -0.414 & 0.048 \\
\hline
\end{tabular}

namely kaempferol, inhibits nitric oxide production and nitric oxide synthase protein expression via HO-1-mediated ROS reduction in murine macrophages (Choi et al., 2012). Although in our study the administration of hydrolyzed and non-hydrolyzed linden extract did not influenced the HO-1 gene expression we were able to identify the 18 genes which were expression targets of HO-1 (Table 4). The Figure 2 presents genes differentially regulated in our study and their relations with HO-1, Nrf2 (NFE2L2) proteins.

The analysis of antioxidant parameters of liver tissue suggests that acid hydrolysis of water extract from linden inflorescence increases the accumulation of quercetin in livers of rats receiving the diet enriched in this extract. The level ofquercetin in this case is more than two-fold higher when compared to rats receiving the diet enriched with non-hydrolyzed extract. This proves the theory that flavonoids in aglycone form and not in glycoside form are preferentially absorbed in small intestine, although the it is glycosides which naturally occur in raw materials of plant origin (McAnlis et al., 1999; Zhao et al., 2011). The increased accumulation of quercetin in the livers of rats receiving hydrolyzed extract resulted in the increase of total antioxidant status of the liver and consequently the decreased fatty acids oxidation in the hepatocytes cell membranes. These results are in accordance with many other studies, which indicated the possibilities of the 
use of biologically active substances of plant origin as a effective strategy for the prevention from the damaging activity of free radicals in intracellular fluids and in cell membranes (Matsukawa et al., 2009; Egert et al., 2008).

Also transcriptomic data clearly show that the non-hydrolyzed linden extract regulates the expression of genes involved in the prevention from free radical oxidative damage and the regeneration after oxidative damage.

\section{Conclusions}

The analysis of oxidative status in rats liver together with the analysis of liver transcriptomic profile suggest that the antioxidant activity of hydrolyzed linden extract is higher that the non-hydrolyzed and occurs on the level of gene expression. However, genes regulated by hydrolyzed linden extract are not only involved in antioxidant status of rat livers but also in immunity and defense, transport, homeostasis and lipid, fatty acid and steroid metabolism.

\section{References}

Aherne S.A., O'Brien N.M., 2002. Dietary flavonols: chemistry, food content, and metabolism. Nutrition. 18, 75-81

Chen C., Kong A.N., 2004. Dietary chemopreventive compounds and ARE/EpRE signaling. Free Radical Biol. Med. 36, 1505-1516

Choi I.S., Choi E.Y., Jin J.Y., Park H.R., Choi J.I., Kim S.J., 2012. Kaempferol inhibits Prevotella intermedia lipopolysaccharideinduced production of nitric oxide through translational regulation in murine macrophages: Critical role of heme oxygenase-1-mediated reactive oxygen species reduction. J. Periodontol. [Epub ahead of print]

Crespy V., Morand C., Besson C., Manach C., Demigne C., Remesy C., 2002. Quercetin, but not its glycosides, is absorbed from the rat stomach. J. Agr. Food Chem. 50, 618-621

Dihal A.A., de Boer V.C., van der Woude H., Tilburgs C., Bruijntjes J.P., Alink G.M., Rietjens I.M., Woutersen R.A., Stierum R.H., 2006. Quercetin, but not its glycosidated conjugate rutin, inhibits azoxymethane-induced colorectal carcinogenesis in F344 rats. J. Nutr. 136, 2862-2867

Egert S., Wolffram S., Bosy-Westphal A., Boesch-Saadatmandi C., Wagner A.E., Frank J., Rimbach G., Mueller M.J., 2008. Daily quercetin supplementation dose-dependently increases plasma quercetin concentrations in healthy humans. J. Nutr. 138: $1615-1621$
Granado-Serrano A.B., Martín M.A., Bravo L., Goya L., Ramos S., 2012. Quercetin modulates Nrf2 and glutathione-related defenses in HepG2 cells: Involvement of p38. Chem.-Biol. Inter. 195, 154-164

Hodek P., Trefil P., Stiborová M., 2002. Flavonoids-potent and versatile biologically active compounds interacting with cytochromes P450. Chem.-Biol. Inter. 139, 1-21

Hong J.T., Yen J.H., Wang L., Lo Y.H., Chen Z.T., Wu M.J., 2009. Regulation of heme oxygenase-1 expression and MAPK pathways in response to kaempferol and rhamnocitrin in PC12 cells. Toxicol. Appl. Pharmacol. 237, 59-68

Kohlmunzer S., 1993. Pharmacognosy: Textbook for students of pharmacy (in Polish). Wydawnictwo Lekarskie PZWL, Warszawa)

Liu S., Hou W., Yao .P, Li N., Zhang B., Hao L., Nüssler A.K., Liu L., 2012. Heme oxygenase-1 mediates the protective role of quercetin against ethanol-induced rat hepatocytes oxidative damage. Toxicol. Vitro 26, 74-80

Manach C., Morand C., Demigné C., Texier O., Régérat F., Rémésy C., 1997. Bioavailability of rutin and quercetin in rats. FEBS Lett. 409, 12-16

Matsukawa N., Matsumoto M., Hara H., 2009. High biliary excretion levels of quercetin metabolites after administration of a quercetin glycoside in conscious bile duct cannulated rats. Biosci, Biotechnol, Biochem, 73, 1863-1875

McAnlis G.T., McEneny J., Pearce J., Young I.S., 1999. Absorption and antioxidant effects of quercetin from onions in man. Eur. J. Clin. Nutr. 53, 92-96

Nakahira K., Takahashi T., Shimizu H., Maeshima K., Uehara K., Fujii H., Nakatsuka H., Yokoyama M., Akagi R., Morita K., 2003. Protective role of heme oxygenase-1 induction in carbon tetrachloride-induced hepatotoxicity. Biochem. Pharmacol. 66, 1091-1105

Sadowska A., 2003. Medicinal plants in veterinary and zootechnics (in Polish). Wydawnictwo SGGW, Warszawa

Smyth G.K., 2004. Linear models and empirical bayes methods for assessing differential expression in microarray experiments. Stat. Appl. Genet. Mol. Biol. 3, 1

Tanigawa S., Fujii M., Hou D.X., 2007. Action of Nrf2 and Keap1 in ARE-mediated NQO1 expression by quercetin. Free Radical Biol. Med. 42, 1690-1703

Weng C.J., Chen M.J., Yeh C.T., Yen G.C., 2011. Hepatoprotection of quercetin against oxidative stress by induction of metallothionein expression through activating MAPK and PI3K pathways and enhancing Nrf2 DNA-binding activity. New Biotechnol. 28, 767-777

Zhao L., Wu J., Yang J., Wei J., Gao W., Guo C., 2011, Dietary quercetin supplementation increases serum antioxidant capacity and alters hepatic gene expression profile in rats. Exp. Biol. Med. 236, 701-706

Zerin T., Kim Y.S., Hong S.Y., Song H.Y., 2012. Quercetin reduces oxidative damage induced by paraquat via modulating expression of antioxidant genes in A549 cells. J. Appl. Toxicol. DOI: 10.1002/jat.2812 